\title{
Average local values and local variances in quantum mechanics
}

\author{
J.G. Muga, J.P. Palao and R. Sala \\ Departamento de Física Fundamental y Experimental, Facultad de Física, Universidad de La \\ Laguna, Tenerife, Spain
}

\begin{abstract}
Several definitions for the average local value and local variance of a quantum observable are examined and compared with their classical counterparts. An explicit way to construct an infinite number of these quantities is provided. It is found that different classical conditions may be satisfied by different definitions, but none of the quantum definitions examined is entirely consistent with all classical requirements.
\end{abstract}

Ref: Physics Letters A 238(1998)90, Electronic version with permission of Elsevier e-mail address: JMUGA@ULL.ES

Fax number: 34-22-603684

PACS 03.65S - Semiclassical theories and applications.

PACS 03.65 - Formalism.

Keywords: Phase space, quantization rules, classical-quantum correspondence, foundations of quantum mechanics. 
Many simple concepts of standard classical statistical mechanics are not easy to translate into quantum mechanics. The average local value and the local spread of a dynamical variable belong to this group. For an ensemble of particles in one dimension characterized by a joint distribution $F(q, p)$ of position $q$ and momentum $p$ the average local value of the dynamical variable $a(q, p)$ is given by

$$
\bar{a}(q)=\frac{1}{P(q)} \iint a\left(q^{\prime}, p\right) \delta\left(q^{\prime}-q\right) F\left(q^{\prime}, p\right) d q^{\prime} d p,
$$

where $P(q) \equiv \int F(q, p) d p$, and the local spread, or local variance, by

$$
\sigma_{a \mid q}^{2}=\overline{a^{2}}(q)-[\bar{a}(q)]^{2}
$$

where $\overline{a^{2}}$ is the local average of the square of $a$, or second order "local moment". Of course an arbitrary power of $a, b(q, p) \equiv a^{n}(q, p)$, is also a function of $(q, p)$, so the expression (1), mutatis mutandis, gives also the local moments of arbitrary order,

$$
\begin{aligned}
\bar{b}(q) & =\frac{1}{P(q)} \iint b\left(q^{\prime}, p\right) \delta\left(q^{\prime}-q\right) F\left(q^{\prime}, p\right) d q^{\prime} d p \\
& =\overline{a^{n}}(q)=\frac{1}{P(q)} \iint a^{n}\left(q^{\prime}, p\right) \delta\left(q^{\prime}-q\right) F\left(q^{\prime}, p\right) d q^{\prime} d p
\end{aligned}
$$

This observation may appear trivial at this point but it will become important when discussing the quantum case. Also, the following marginal, joint, and conditional probabilities can be defined,

$$
\begin{aligned}
P(a) & =\iint \delta[a-a(q, p)] F(q, p) d q d p \\
P(a, q) & =\iint \delta\left[a-a\left(q^{\prime}, p\right)\right] \delta\left(q^{\prime}-q\right) F\left(q^{\prime}, p\right) d q^{\prime} d p \\
P(a \mid q) & =P(a, q) / P(q)
\end{aligned}
$$

where the last equation is simply Bayes' rule. In terms of these later quantities $\bar{a}$ and $\sigma_{a \mid q}^{2}$ take the form

$$
\bar{a}(q)=\int a P(a \mid q) d a=\frac{1}{P(q)} \int a P(a, q) d a
$$

and

$$
\sigma_{a \mid q}^{2}=\frac{1}{P(q)} \int(a-\bar{a})^{2} P(a, q) d a .
$$

The total (or global) average is simply the "q-average" [i.e. an average over $q$ of a $q$-dependent function weighted by $P(q)$ ] of the local averages,

$$
\langle a\rangle=\iint a(q, p) F(q, p) d q d p=\int \bar{a}(q) P(q) d q,
$$


whereas the total variance takes the form,

$$
\begin{aligned}
\sigma_{a}^{2} & \equiv \iint(a-\langle a\rangle)^{2} P(a, q) d a d q \\
& =\int \sigma_{a \mid q}^{2} P(q) d q+\int(\bar{a}(q)-\langle a\rangle)^{2} P(q) d q
\end{aligned}
$$

$\sigma_{a}^{2}$ is the $q$-average of the local variances plus the " $q$-variance" of the local averages. This is an appealing decomposition, and it seems reasonable to seek for definitions of quantum local moments that preserve its structure.

In a recent publication in this journal, L. Cohen [1] has argued that it is natural to interpret the following quantities as the local value of the observable associated with the hermitian operator $\widehat{A}$ and its local spread,

$$
\begin{aligned}
\bar{A}^{S}(q) & \equiv\left(\frac{\langle q|\widehat{A}| \psi\rangle}{\langle q \mid \psi\rangle}\right)_{\mathcal{R}} \\
{\left[\sigma_{A \mid q}^{2}\right]^{C} } & \equiv\left(\frac{\langle q|\widehat{A}| \psi\rangle}{\langle q \mid \psi\rangle}\right)_{\mathcal{I}}^{2} .
\end{aligned}
$$

The superscripts $S$ and $C$ will distinguish these quantities from others to be defined later (the reason for using different letters will become clear soon), and the subscripts $\mathcal{R}$ and $\mathcal{I}$ indicate "real" and "imaginary" parts. (This interpretation of (13) has also been proposed in [24].) These quantities obey a relation with the form of Eq. (12),

$$
\sigma_{A}^{2}=\int \sigma_{A \mid q}^{2}|\psi(q)|^{2} d q+\int\left(\bar{A}^{S}(q)-\langle\widehat{A}\rangle\right)^{2}|\psi(q)|^{2} d q
$$

and the analogy with the classical equation has been invoked to understand the significance of the two terms in (15) [1]. In this letter we shall explore how far the classical-quantum analogy goes. We shall see in particular that the definitions (13) and (14) do not always lead to results consistent with the form of equations (2) and (3). To this end it is useful to introduce an operator that symmetrizes $\widehat{A}$ and $\delta(\widehat{q}-q)=|q\rangle\langle q|$,

$$
\widehat{A_{q}}=\frac{1}{2}[\widehat{A} \delta(\widehat{q}-q)+\delta(\widehat{q}-q) \widehat{A}]
$$

and express (13) in terms of it as,

$$
\bar{A}^{S}(q)=\frac{\left\langle\widehat{A_{q}}\right\rangle}{\varrho(q)} .
$$

where $\varrho(q)=|\psi(q)|^{2}$ is the probability density and $\left\langle\widehat{A_{q}}\right\rangle \equiv\left\langle\psi\left|\widehat{A_{q}}\right| \psi\right\rangle$. Note that the expectation value of $\widehat{A}_{q}$ is a "local density of $A$ ", i.e., a quantity that integrated over $q$ provides the global average,

$$
\int\left\langle\psi\left|\widehat{A_{q}}\right| \psi\right\rangle d q=\langle\widehat{A}\rangle
$$


(We shall see below that other definitions also satisfy this condition.) For consistency, the definition for the local average should apply to any operator, and in particular to $\widehat{A}^{2}$. The local average of $\widehat{A}^{2}$ is accordingly given by

$$
{\overline{A^{2}}}^{S}(q)=\frac{\left\langle\left(\widehat{A}^{2}\right)_{q}\right\rangle}{\varrho(q)}=\frac{1}{2 \varrho(q)}\left\langle\psi\left|\left[\widehat{A}^{2} \delta(\widehat{q}-q)+\delta(\widehat{q}-q) \widehat{A}^{2}\right]\right| \psi\right\rangle .
$$

Following (2), a quantum local variance for $\widehat{A}$ is "naturally" defined as

$$
\begin{aligned}
{\left[\sigma_{A \mid q}^{2}\right]^{S} } & =\bar{A}^{2}(q)-\left[\bar{A}^{S}(q)\right]^{2} \\
& =\frac{1}{2 \varrho(q)}\left\langle\psi\left|\left[\widehat{A}^{2} \delta(\widehat{q}-q)+\delta(\widehat{q}-q) \widehat{A}^{2}\right]\right| \psi\right\rangle-\left[\bar{A}^{S}(q)\right]^{2}
\end{aligned}
$$

where the superscript $S$ reminds that only symmetrized operators are used. This definition for the local variance also satisfies the decomposition (12), but it is different from (14). To see this in more detail let us write (14) as

$$
\begin{aligned}
{\left[\sigma_{A \mid q}^{2}\right]^{C} } & =\left(\frac{\langle q|\widehat{A}| \psi\rangle}{\langle q \mid \psi\rangle}\right)_{\mathcal{I}}^{2}=\left|\frac{\langle q|\widehat{A}| \psi\rangle}{\langle q \mid \psi\rangle}\right|^{2}-\left(\frac{\langle q|\widehat{A}| \psi\rangle}{\langle q \mid \psi\rangle}\right)_{\mathcal{R}}^{2} \\
& =\frac{1}{\varrho(q)}\langle\psi|\widehat{A} \delta(\widehat{q}-q) \widehat{A}| \psi\rangle-\left[\bar{A}^{S}(q)\right]^{2}
\end{aligned}
$$

Clearly the two procedures imply two different interpretations of the local density for the square of $\widehat{A}$. In general,

$$
\langle\psi|\widehat{A} \delta(\widehat{q}-q) \widehat{A}| \psi\rangle \neq \frac{1}{2}\left\langle\psi\left|\left[\widehat{A}^{2} \delta(\widehat{q}-q)+\delta(\widehat{q}-q) \widehat{A}^{2}\right]\right| \psi\right\rangle .
$$

However their integrals over $q$ are both equal to $\left\langle\widehat{A}^{2}\right\rangle$. Eq. (20) provides a local variance consistent with the definition given for the local average and it is in agreement with the classical expressions (12) and (2), but it is not semidefinite positive. Its literal interpretation as a variance is therefore impossible. This resembles the status of the Wigner function and other phase space quasi-distribution functions. While not interpretable as probability distributions they can be used to correctly evaluate expectation values and to investigate the classical limit in a classical-like phase space language.

In fact the decompositions of the total variance compatible with (12) and (2) are infinite. They can be found by means of the phase space formalisms described within the general framework provided also by Cohen [5,6]. Each of these formalisms is associated with a particular function $f(\theta, \tau)$ of auxiliary variables $\theta$ and $\tau$. The density operator and the operator $\widehat{A}$ are related, respectively, to a quasi distribution function $F(q, p ;[f])$ and a phase space representation $\tilde{A}(q, p ;[f])$ [both depend functionally on $f$; Note that $\tilde{A}(q, p ;[f])$ is not necessarily equal to the classical function $a(q, p)]$ in such a way that the expectation value of $\widehat{A}$ is given by the phase space integral, 
$\langle\widehat{A}\rangle=\iint \tilde{A}(q, p ;[f]) F(q, p ;[f]) d q d p$. These formalisms are also closely related to the "quantization rules", that define mappings from phase space functions to operators. The rules are generally used to associate a quantum operator with the classical function $a(q, p)$.

Local values can be defined using any of these phase space formalisms, see the classical expression (8), as

$$
\bar{A}(q ;[f])=\frac{1}{\varrho(q)} \int \tilde{A}(q, p ;[f]) F(q, p ;[f]) d p .
$$

Their $q$-average is the global average, $\langle\widehat{A}\rangle=\int \bar{A} \varrho d q$, and the variance can be decomposed in agreement with (12) by writing

$$
\begin{aligned}
\sigma_{A}^{2} & =\int\left[\int \frac{\widetilde{A^{2}}(q, p ;[f]) F(q, p ;[f])}{\varrho(q)} d p\right] \varrho(q) d q-\int \bar{A}^{2}(q ;[f]) \varrho(q) d q \\
& +\int \bar{A}^{2}(q ;[f]) \varrho(q) d q-\langle\widehat{A}\rangle^{2},
\end{aligned}
$$

where $\widetilde{A^{2}}(q, p ;[f])$ is the phase space representation of $\widehat{A}^{2}$.

The first two terms in (25) may be regarded as the $q$-average of the "local variance"

$$
\sigma_{A \mid q}^{2}[f] \equiv \overline{A^{2}}(q ;[f])-\bar{A}^{2}(q ;[f]),
$$

while the last two terms take the form of a $q$-variance of local averages. An important point is that $\widetilde{A^{2}}(q, p ;[f])$ is in general different from the square $\tilde{A}^{2}(q, p ;[f])$ so it is not necessarily a positive function. Unless $f$ is suitably chosen $F$ is not positive either, so that, contrary to the classical local variance, and in spite of the "square" used in the notation, $\sigma_{A \mid q}^{2}[f]$ is not necessarily positive. Of course for a given kernel $f, \sigma_{A \mid q}^{2}[f]$ can be positive for particular observables and, similarly, for a given observable a family of kernels will give a positive local variances. In the case of momentum, $\widehat{A}=\widehat{p}$, Cohen has noted that (26) is positive for a family of kernel functions $f$ that lead precisely to the choices (13) and (14) [7]. In general, however, each observable requires a different analysis.

We shall next elaborate on the momentum observable and its powers using two of these formalisms because of their close relation to the previously discussed choices and to classical expressions. The Rivier-Margeneau-Hill (RMH) formalism is obtained by taking $f=\cos (\theta \tau \hbar / 2)$ [8, 8, 5,6]. We shall denote the corresponding representation of the state, namely the Margeneau-Hill function, as $F^{M H}$. The subscript $M H$ will be also used for the local averages and variances, (24) and (26), defined within this framework. The corresponding mapping from phase space representatives to operators is the "Rivier rule" [8]. When applied to a phase space function with the factorized form $g(q) h(p)$ it gives the symmetrized operator $[g(\widehat{q}) h(\widehat{p})+h(\widehat{p}) g(\widehat{q})] / 2$. Note that for an arbitrary product of 
phase space functions $A B$, this symmetrization is not always equal to the simple symmetrization rule $(\widehat{A} \widehat{B}+\widehat{B} \widehat{A}) / 2$, where $\widehat{A}$ and $\widehat{B}$ are quantum operators assigned to $A$ and $B$ by some prescription (that could be in fact Rivier's rule), see e.g. [10]. However, if $A(q)$ and $B(p)$ are, respectively, functions of $q$ and $p$ only, and the associated operators are $A(\widehat{q})$ and $B(\widehat{p})$, then the two symmetrization procedures agree. This implies in particular that ${\overline{p^{n}}}^{S}(q)={\overline{p^{n}}}^{M H}(q)$ and $\left[\sigma_{p \mid q}^{2}\right]^{S}=\left[\sigma_{p \mid q}^{2}\right]^{M H}$.

It has been argued that the interpretation of (13) as the local average leads to the MargeneauHill function [四]. This connection can indeed be made, but, remarkably, the local values obtained by means of the RMH formalism in general differ from (13). To understand how this may happen let us briefly review the derivation of the Margeneau-Hill function in [4]. The idea is to define a conditional probability density $P^{S}(p \mid q)$ making use of the characteristic function concept, i.e., by inverting

$$
\begin{gathered}
G(\tau, q) \equiv \overline{e^{i \tau p}} S(q)=\int e^{i \tau p} P^{S}(p \mid q) d p \\
P^{S}(p \mid q) \equiv \frac{1}{2 \pi} \int e^{-i \tau p} \overline{e^{i \tau p} S}(q) d \tau
\end{gathered}
$$

Expanding the exponential in ${\overline{e^{i \tau p}}}^{S}$, there results a series where the coefficients are the local averages of the powers of $p,{\overline{p^{n}}}^{S}$. Using the coordinate representation for the momentum operator the Taylor series for $\psi(q \pm \hbar \tau)$ can be recognized,

$$
\bar{e}^{i \tau p} S(q)=\sum_{n} \frac{(i \tau)^{n}}{n !} \bar{p}^{n} S(q)=\frac{\psi(q+\hbar \tau)}{2 \psi(q)}+\frac{\psi^{*}(q-\hbar \tau)}{2 \psi^{*}(q)} .
$$

If in addition one defines a quasi-joint probability distribution by the product $\varrho(q) P^{S}(p \mid q)$, i.e., by formally adopting the structure of Bayes' theorem, this quasi-probability turns out to be the Margeneau-Hill function,

$$
F^{M H}(q, p)=[\langle p \mid \psi\rangle\langle\psi \mid q\rangle\langle q \mid p\rangle]_{\mathcal{R}}
$$

But, as discussed before, in general $\bar{A}^{S} \neq \bar{A}^{M H}$. The symmetrized operator $\left(\widehat{A}^{2}\right)_{q}$ is equal to the one provided by Rivier's rule if $A$ is only a function of $p$, but will differ in general. The use of the structure of Bayes' theorem in the derivation of (30) is the key point that explains this seeming contradiction. This is a theorem valid for actual probabilities, but $P^{S}(p \mid q)$ and $F^{M H}(q, p)$ are not. In fact they can be negative.

Finally, the comparison with the classical equations satisfied by local average and the variance is completed here by studying the time dependence, in particular the equations for time derivatives of the local averages of the powers of $p$, i.e., the equations of hydrodynamics. In this context the WeylWigner (WW) phase space formalism is privileged. This formalism is associated with the simplest 
choice, $f=1$ [11,5:6]. The phase space representative of the state is the Wigner function, $F^{W}(q, p)$, and the corresponding rule is the Weyl quantization rule. A superscript $W$ will denote the local quantities (24) and (26) calculated with this formalism.

Cohen's prescription, Eq. (13), and the phase space formalisms RMH and WW lead to the same equation for the local density, namely the continuity equation,

$$
\frac{\partial \varrho(q)}{\partial t}=-\frac{\partial}{\partial q}[\varrho(q) \bar{p}(q)]
$$

where $\bar{p}=\bar{p}^{S}=\bar{p}^{M H}=\bar{p}^{W}$. For the first local moment, the Wigner function leads to an "equation of motion" with exactly the same form as the classical one 12],

$$
\frac{\partial \bar{p}^{W}}{\partial t}=-\frac{\bar{p}^{W}}{m} \frac{\partial \bar{p}^{W}}{\partial q}-\frac{\partial V(q)}{\partial q}-\frac{1}{m \varrho(q)} \frac{\partial\left(\varrho(q)\left[\sigma_{p \mid q}^{2}\right]^{W}\right)}{\partial q}
$$

$m$ being the mass, and $V(q)$ the potential function. (Even though the form is equal, the numerical values differ in general between the classical and the quantum cases, and the local variance $\left[\sigma_{p \mid q}^{2}\right]^{W}$ is not semidefinite positive [12].) However, in the other approaches the local variances are different,

$$
\begin{aligned}
{\left[\sigma_{p \mid q}^{2}\right]^{W} } & =\left[\sigma_{p \mid q}^{2}\right]^{M H}+\frac{1}{4 \varrho(q)}\langle 2 \widehat{p} \delta(\widehat{q}-q) \widehat{p}-\widehat{p} \delta(\widehat{q}-q)-\delta(\widehat{q}-q) \widehat{p}\rangle \\
& =\left[\sigma_{p \mid q}^{2}\right]^{C}-\frac{1}{4 \varrho(q)}\langle 2 \widehat{p} \delta(\widehat{q}-q) \widehat{p}-\widehat{p} \delta(\widehat{q}-q)-\delta(\widehat{q}-q) \widehat{p}\rangle,
\end{aligned}
$$

and there appear extra terms which are not present in the classical equation (for the RMH formalism they were studied by Sonego [13]). At least for several model potentials studied in ref. 114 the WW formalism is not only closer to classical mechanics formally in this context; it is also numerically closer. The local kinetic energy densities $\varrho(q){\overline{p^{2}}}^{W}(q) /(2 m), \varrho(q){\overline{p^{2}}}^{M H}(q) /(2 m)$, and $\langle\widehat{p} \delta(\widehat{q}-q) \widehat{p}\rangle /(2 m)$ were evaluated, and the one provided by the WW formalism was clearly the closest (numerically) to the classical values [14. Determining the extent of this agreement is an interesting open question for a separate study.

In summary, we have examined different definitions of "quantum local averages and variances", and their similarities and differences with various classical expressions. None of them satisfies all the relations valid classically. It is useful to maximize the agreement with classical mechanics for examining the classical limit, and to gain physical insight in certain applications, but it should be noted that different classical criteria are satisfied by different quantum definitions. For specific applications one of the definitions may turn out to be the most convenient, but in fact each of them contains a piece of information which is only partially analogous to the corresponding classical quantity. 


\section{Acknowledgments}

We acknowledge the referee for very interesting comments. Support by Gobierno Autónomo de

Canarias (Spain) (Grant PI2/95) and by Ministerio de Educación y Ciencia (Spain) (PB 93-0578) is acknowledged. JPP acknowledges an FPI fellowship from Ministerio de Educación y Ciencia. 


\section{References}

1. L. Cohen, Phys. Lett. A 212 (1996) 315

2. P. R. Holland, The quantum theory of motion (Cambridge Univ. Press, Cambridge, 1993)

3. K. K. Wan and P. Summer, Phys. Lett. A 128 (1988) 458

4. R. I. Sutherland, J. Math. Phys. 23 (1982) 2389

5. L. Cohen, J. Math. Phys. 7 (1966) 781.

6. R. Sala, J. P. Palao and J. G. Muga, Phys. Lett. A, accepted

7. L. Cohen, Found. Phys. 20 (1990) 1455; L. Cohen, Time-Frequency analysis (Prentice-Hall, Englewood Cliffs, New Jersey, 1995)

8. D. C. Rivier, Phys. Rev. 83 (1957) 862.

9. H. Margenau and R. N. Hill, Progr. Theoret. Phys. (Kioto) 26 (1961) 722; G. C. Summerfield and P. F. Zweifel, J. Math. Phys. 10 (1969) 233

10. E. T. García Álvarez and A. D. González, Am. J. Phys. 59 (1991) 279

11. E. Wigner, Phys. Rev. A 40 (1932) 749; J. E. Moyal, Proc. Cambridge Philos. Soc. 45 (1949) 99; H. Weyl, The Theory of Groups and Quantum Mechanics (Dover, New York, 1950)

12. J. G. Muga, R. Sala and R. F. Snider, Physica Scripta 47 (1993) 732

13. S. Sonego, Phys. Rev. A 42 (1990) 3733

14. C. C. Real, J. G. Muga and S. Brouard, Am. J. Phys. 65 (1997) 157 